\title{
Necroptosis as a potential therapeutic target in multiple organ dysfunction syndrome
}

\author{
Yao-Li Cui ${ }^{1,2}$, Li-Hua Qiu ${ }^{1}$, Shi-Yong Zhou ${ }^{1}$, Lan-Fang Li ${ }^{1}$, Zheng-Zi Qian ${ }^{1}$, Xian- \\ Ming Liu ${ }^{1}$, Hui-Lai Zhang ${ }^{1}$, Xiu-Bao Ren ${ }^{3}$ and Yong-Qiang Wang ${ }^{2}$ \\ ${ }^{1}$ Department of Lymphoma, Tianjin's Clinical Research Center for Cancer and Key Laboratory of Cancer Prevention and \\ Therapy, Tianjin Medical University Cancer Institute \& Hospital, National Clinical Research Center for Cancer, Tianjin 300060, \\ China \\ ${ }^{2}$ Department of Intensive Care Unit and Key Laboratory for Critical Care Medicine of The Ministry of Health, Emergency \\ Medicine Research Institute, Tianjin First Center Hospital, Tianjin 300192, China \\ ${ }^{3}$ Department of Biotherapy, Key Laboratory of Cancer Immunology and Biotherapy and Key Laboratory of Cancer Prevention \\ and Therapy, Tianjin's Clinical Research Center for Cancer, Tianjin Medical University Cancer Institute \& Hospital, National \\ Clinical Research Center for Cancer, Tianjin 300060, China \\ Correspondence to: Hui-Lai Zhang, email: zhlwgq@126.com \\ Xiu-Bao Ren, email: renxiubao1111@163.com \\ Yong-Qiang Wang, email: wangyongqiang1100@163.com \\ Keywords: multiple organ dysfunction syndrome, necroptosis, necrosome, high-mobility group box 1, mixed-lineage kinase \\ domain-like \\ Received: March 24, $2017 \quad$ Accepted: April 17, $2017 \quad$ Published: May 29, 2017 \\ Copyright: Cui et al. This is an open-access article distributed under the terms of the Creative Commons Attribution License 3.0 \\ (CC BY 3.0), which permits unrestricted use, distribution, and reproduction in any medium, provided the original author and source \\ are credited.
}

\section{ABSTRACT}

Purpose: To investigate how necroptosisis, i.e. programmed necrosis, is involved in MODS, and to examine whether Nec-1, a specific necroptosis inhibitor, ameliorates multiorgan injury in MODS.

Experimental Design: A model of MODS was established in six-week old SD rats using fracture trauma followed by hemorrhage. Control animals received sham surgery. Cell death form and necrosome formation were measured by fluorescenceactivated cell sorting and western blotting. MODS rats were randomly assigned to receive $\mathrm{Nec}-1$ or saline with pretreatment and once daily. The first end-point was 72 hours survival. Organ injury and dysfunction, inflammatory cytokine levels, and necroptotic execution protein expression were also recorded.

Results: Organ injury and dysfunction were significantly more severe in the MODS group than the sham group (all $p<0.01$ ). Furthermore, MODS-induced liver, lung and kidney tissue injury was characterized by necroptosis rather than apoptosis, and accompanied by necrosome formation. Compared to MODS group, Nec-1 administration significantly improved 72 hours survival $(p<0.01)$. Nec-1 administration significantly reduced necroptosis-induced liver, lung and kidney injury and dysfunction, inhibited inflammatory cytokines production, inhibited release of necroptotic execution proteins such as high-mobility group box 1 and mixed-lineage kinase domain-like protein pseudokinase in MODS rats (all $p<0.01$ ).

Conclusions: These results suggest that necroptosis is involved the pathology of MODS. Further, a necroptotic inhibitor Nec-1 may be considered as an adjunct treatment for MODS.

\section{INTRODUCTION}

MODS describes the progressive dysfunction of two or more organ systems following an acute threat to systemic homeostasis. MODS is the leading cause of mortality in critically ill patients [1], and in most cases occurs secondary to severe sepsis or septic shock, trauma, neoplastic diseases, or SIRS [2]. To improve the prognosis of this disease, the 
pathological and physiological processes comprising MODS have been investigated, however, its complex multifactorial pathology remains poorly understood [3-5].

In 1991, a joint conference committee of the American College of Chest Physicians and the Society of Critical Care Medicine described SIRS and the corresponding compensatory anti-inflammatory responses [6] in which a cascade of effects arise from an imbalance between pro- and anti-inflammatory mediators, leading to the development of MODS [7-9]. However, no effective treatments or preventive measures have been found to successfully target inflammation in MODS. Therefore, understanding the molecular mechanisms underpinning this syndrome may inform potential clinical treatment strategies.

Necrosis has been considered a non-programmed form of cellular injury and premature death; however, it was recently recognized that one form of cell death morphologically classified as necrosis could also be regulated in a programmed manner via defined signal transduction pathways. This process was termed necroptosis, which is dependent RIP1, RIP3, and a MLKL form a multiprotein complex called a necrosome [10]. Accumulating evidence indicates that necroptosis is involved in the regulation of lethal SIRS [11-14], ischemia-reperfusion injury [15], and traumatic injury [16]. Therefore, elucidating the molecular mechanisms underpinning necroptosis may inform development of novel treatment strategies for MODS.

In recent years small molecule necroptosis inhibitors have proved to be both effective tools with which to study cell death, and to have the potential to serve as therapeutic agents for necroptotic diseases. For example, Nec-1 was reported as the first inhibitor of necroptosis, and exhibited therapeutic potential [17-18]. In this study, we found that necroptosis is involved the pathologic process of MODS, and further that Nec-1 may represent a clinically efficacious adjunct treatment in MODS.

\section{RESULTS}

\section{Successful establishment of MODS rat model}

A SD rat model of MODS was successfully established using fracture trauma followed by hemorrhage. MODS was manifested in significantly more severe multiorgan injury than sham surgery at 12 hours (Figure $1 \mathrm{~A}$ and $1 \mathrm{~B})$. In the MODS group, levels of multiorgan dysfunction markers were significantly higher than in the sham group at 12 hours (all $p<0.01$ ). Furthermore, the 24 hours survival rate in the MODS group was $60 \%$, while no animals in the sham group died $(p<0.01)$.

\section{MODS-induced multiorgan necroptosis dominates over apoptosis}

In order to determine which form of cell death was involved in MODS, we measured the percentage of apoptotic and necroptotic (necrotic) cells at 12 hours after MODS using flow cytometry. We found that in MODS necroptosis dominates over apoptosis in liver, lung, and kidney at 12 hours (Figure 2, all $p<0.05$ ).

\section{Necrosome formation involved in MODS- induced necroptosis}

To investigate the expression of components of the necrosome in MODS, we measured RIP1, RIP3, and MLKL expression in the liver, lung, and kidney of MODS model and sham rats. We found that RIP1, RIP3 and MLKL were expressed significantly more highly in the liver, lung and kidney of MODS animals than sham-group animals in a time-dependent, while RIP1 expression did not difference significantly between two groups in the lung (all $p<0.05$, Figure 3A-3C).

\section{Nec-1 administration improves the prognosis of rats with MODS}

Seventy-two hours after surgery, all sham group animals survived, while the survival rate in the MODS group was only $65 \%$ at 24 hours and $35 \%$ at 48 hours after surgery. Log-rank analysis of the 72 hours survival curves demonstrated that administration of Nec-1 improved the survival rate in the MODS group to $85 \%$ at 24 hours and $80 \%$ at 72 hours after surgery (all $p<0.01$, Figure 4 ).

\section{Nec-1 administration reduces necroptosis- induced liver injury}

No liver cell injury was detected in the sham group; however, rats with MODS presented with exhibited albuminoid degeneration and vacuolar degeneration in the liver in a time-dependent. Locally, eosinophilic variants and spotty liver necrosis were observed; phepatic sinus Kupffer cell proliferation and further inflammatory cell infiltration were also identified in the liver. However, only focal eosinophilic variants of liver cells were observed in the Nec-1 treatment group (Figure 5A).

These results were confirmed by assessment of liver function. In the Nec-1 treatment group, the levels of ALT and AST were significantly lower than in the MODS group at 4 hours and 8 hours (all $p<0.01$, Figure $5 \mathrm{~B}$ and $5 \mathrm{C}$ ). In addition, in Nec-1-treated animals, levels of the inflammatory cytokines TNF- $\alpha$ and IL- $1 \beta$ were significantly lower than in the MODS group at 4 hours and 8 hours (all $p<0.05$, Figure 5D and 5E).

\section{Nec-1 administration reduces necroptosis- induced lung injury}

Similarly, no lung cell injury was observed in the sham group; while MODS rats exhibited alveolar edema complicated with hemorrhage and pulmonary interstitial edema complicated with focal inflammatory cell infiltration in a time-dependent. Alveolar septum 
thickening and interstitial pulmonary perivascular lymphocytic infiltration were also observed. In the Nec-1 treatment group, the lesions were markedly less pronounced, and pulmonary interstitial infiltration, and alveolar septum inflammatory cell infiltration were only occasionally visible (Figure 5F). Furthermore, Nec1 treated animals had lower BALF, TNF- $\alpha$, and IL- $1 \beta$ than MODS group animals at 12 hours and 24 hours (all $p<0.05$, Figure 5G-5I).

\section{Nec-1 administration reduces necroptosis- induced kidney injury}

No kidney cell injury was observed in the sham group, while in the MODS group hyperemic and ischemic changes were observed in the glomeruli. Renal tubular epithelial cells exhibited albuminoid degeneration, and granular degeneration, and partial epithelial vacuolar degeneration was observed. Local interstitial perivascular
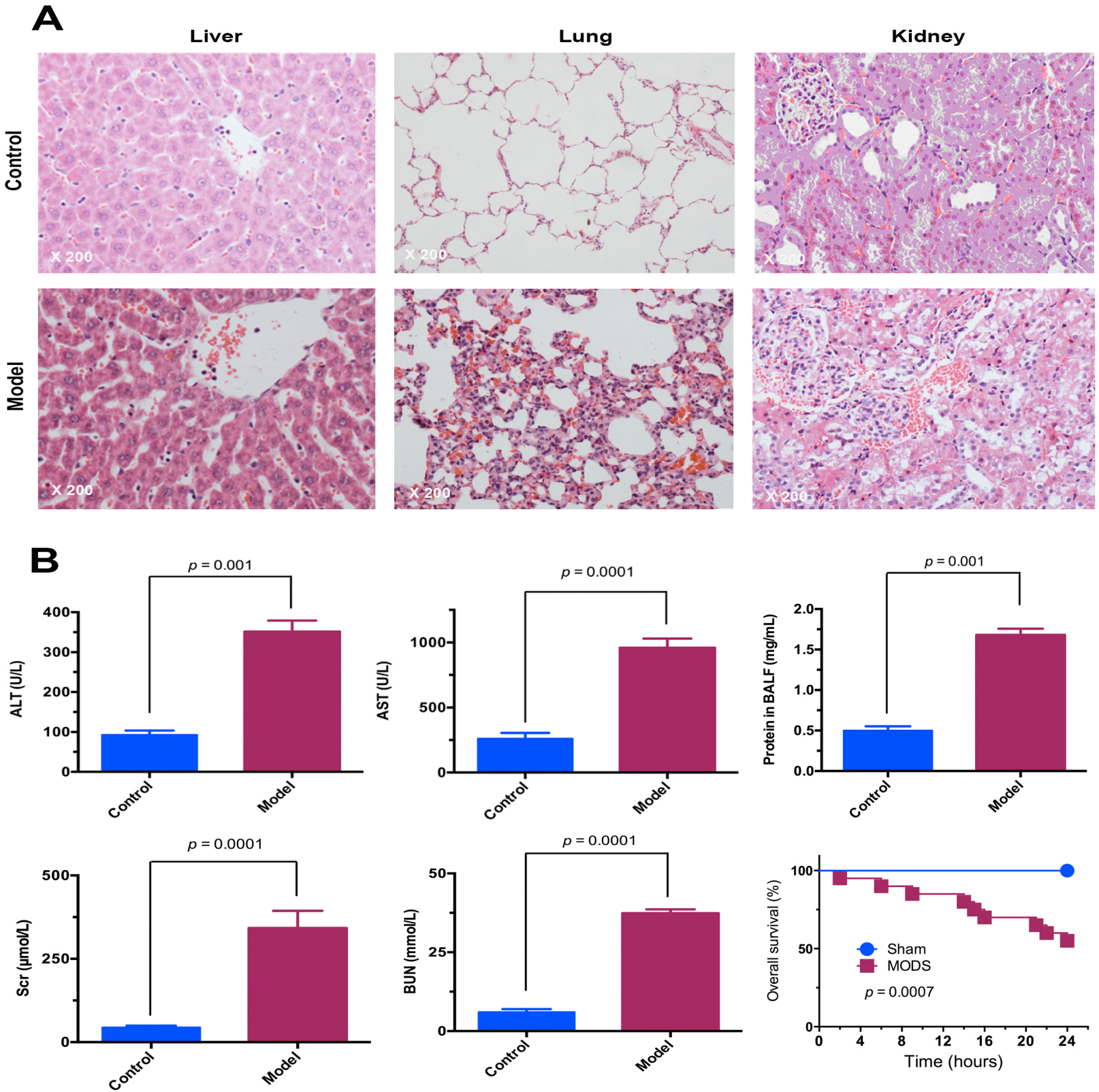

Figure 1: The MODS rat model was successfully established. (A) Pathological changes in the liver, lung, and kidney of MODS and sham group rats. Tissues were stained with HE and observed under light microscope (magnification, $\times 200$ ). (B) Multiorgan function marker levels in MODS and sham groups: liver function markers; lung function marker; kidney function markers, and survival of MODS and sham group rats 24 hours. 


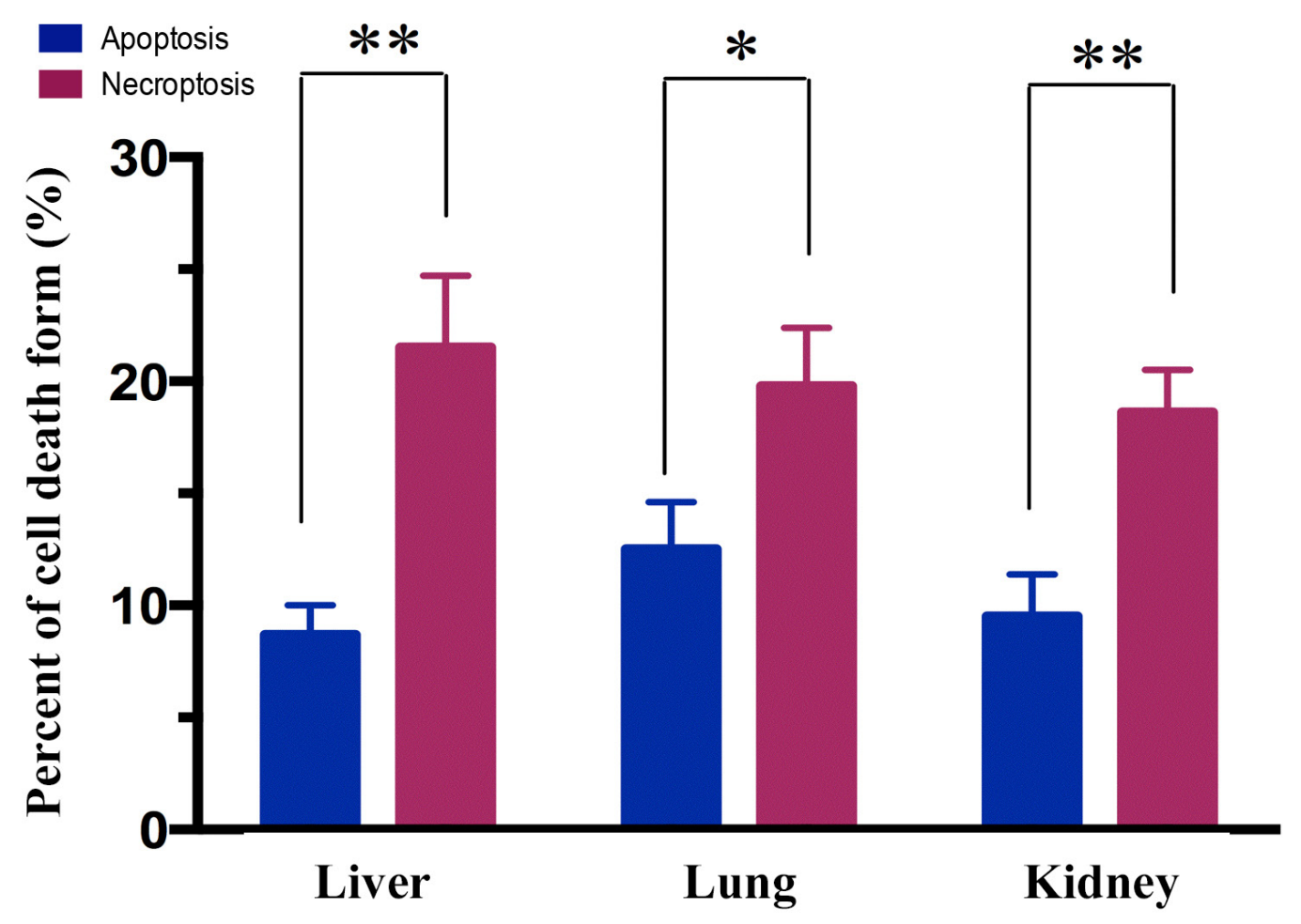

Figure 2: Necroptosis dominates over apoptosis in MODS rats. Rats from MODS group was sacrificed after inoculation; their tissues were obtained. Annexin V/PI staining was assessed by flow cytometry to assess the form of cell death in multiorgan. Apoptosis: Annexin-V positive cells; necrosis: PI positive and Annexin-V negative cells (mean \pm standard deviation). ${ }^{*} p<0.05,{ }^{* *} p<0.01$.
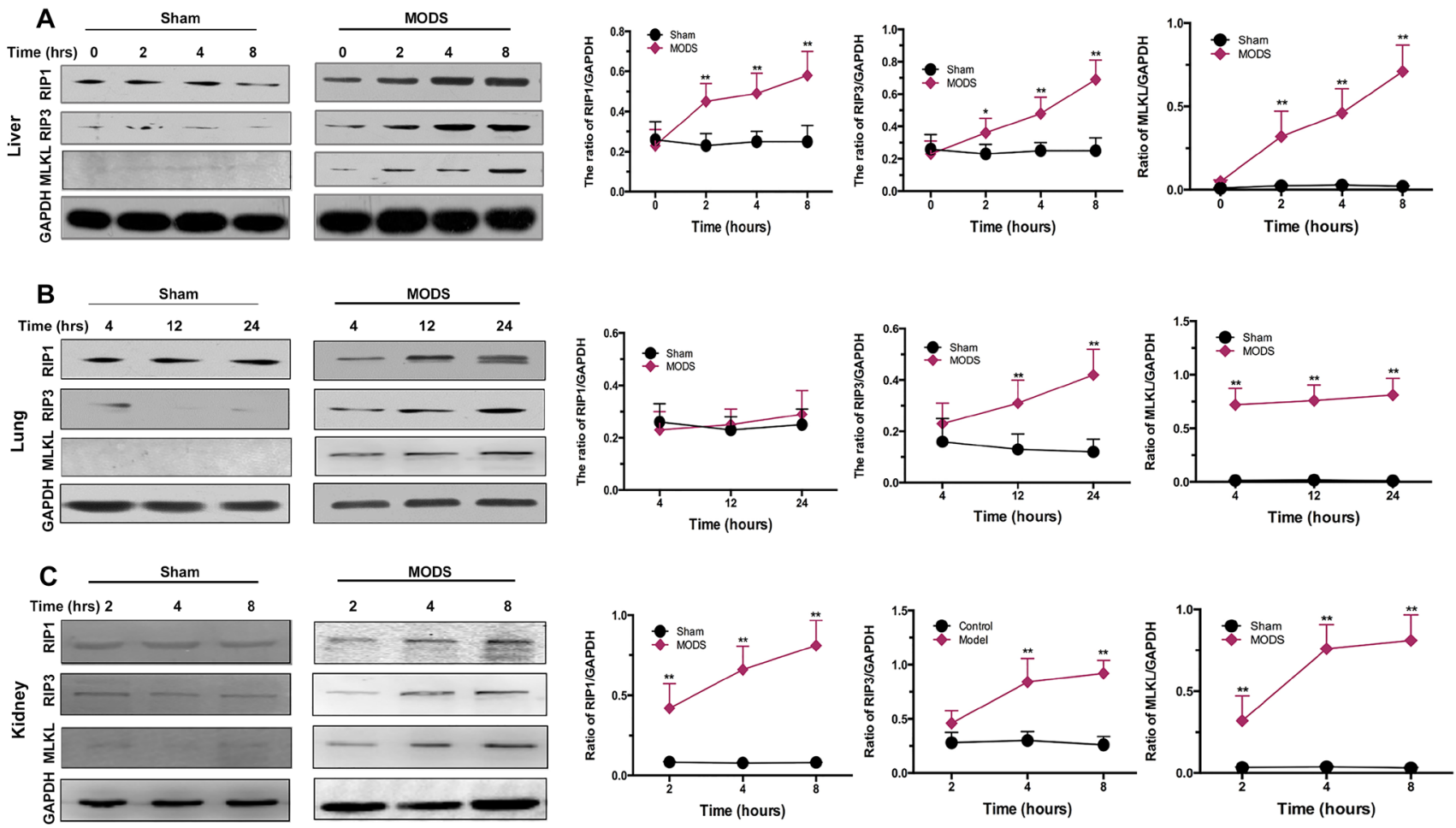

Figure 3: Necrosome formation in MODS-induced necroptosis. Rats from the sham and MODS groups were sacrificed; organ tissues were obtained. (A-C) Expression of RIP1, RIP3, and MLKL protein was evaluated by western blotting in liver, lung, and kidney. Relative protein expression levels were calculated relative to GAPDH. Data are expressed as mean \pm standard deviation. $* p<0.05$ vs. the sham group, $* * p<0.01$ vs. the sham group. 
edema and lymphocyte infiltration were also observed in a time-dependent. In the Nec-1 treatment group, only partial glomerular hyperemia and local renal tubular epithelial albuminoid degeneration were visible (Figure 5J).

These results were confirmed by assessment of kidney function. In the Nec-1 treatment group the tubular necrotic score, and levels of BUN, and SCR were significantly lower than in the MODS group at 12 hours and 24 hours (all $p<0.01$, Figure $5 \mathrm{~K}-5 \mathrm{M}$ ). Taken together, these results clearly demonstrate that $\mathrm{Nec}-1$ reduced MODS-induced multiorgan injury and dysfunction.

\section{Nec-1 inhibits HMGB1 release and MLKL activation}

To investigate the mechanism by which Nec-1 ameliorated multiorgan injury and dysfunction in MODS, we examined the effect of Nec-1 on critical substrates of necrosome formation; HMGB1 release and MLKLpseudokinase ( $\mathrm{p}-\mathrm{MLKL}$ ).

In sham rats, HMGB1 was predominantly located in the nucleus, whereas in MODS rats, higher amounts of HMGB1 were found to be translocated from the nucleus to the cytoplasm. Nec-1 significantly suppressed HMGB1 translocation from the nucleus to the cytoplasm at 12 hours (Figure 6A and 6C). Similarly, in MODS, release of HMGB1 into the serum was increased, while Nec-1 significantly suppressed HMGB1 release in MODS rats at 8 hours $(p<0.05$, Figure 6B).

In addition, we found that p-MLKL was strongly induced in MODS rats, while Nec-1 significantly suppressed p-MLKL in MODS rats at 12 hours $(p<0.05$, Figure 6D). These data suggest that Nec-1 treatment significantly inhibited HMGB1 release and MLKL pseudokinase.

\section{DISCUSSION}

Despite significant improvements in the medical management of MODS, it is still associated with a high mortality rate. To our knowledge, this is the first report of the role of necroptosis in MODS. In the present study, our results indicate that necroptosis is involved the pathology of MODS, and Nec-1 exerted a marked treatment effect by attenuating multiorgan injury and dysfunction, significantly improving survival of MODS rats. This treatment effect was due primarily to suppression of necroptotic execution protein activation and inflammatory cytokine production. Therefore, a necroptotic inhibitor Nec-1 may be considered as an adjunct treatment in MODS.

Accumulating evidence indicates that necroptosis is involved in the regulation of lethal SIRS [11-14]. In this study, MODS-induced multiorgan necroptosis exceeded apoptosis, and was accompanied by necrosome formation. Necroptosis can be executed by necrosomes including RIP1, RIP3, and MLKL [19]. We found that RIP1, RIP3, and MLKL were expressed significantly more highly in the liver, lung and kidney of MODS animals than shamgroup animals, while RIP1 expression did not differ significantly between two groups in the lung.

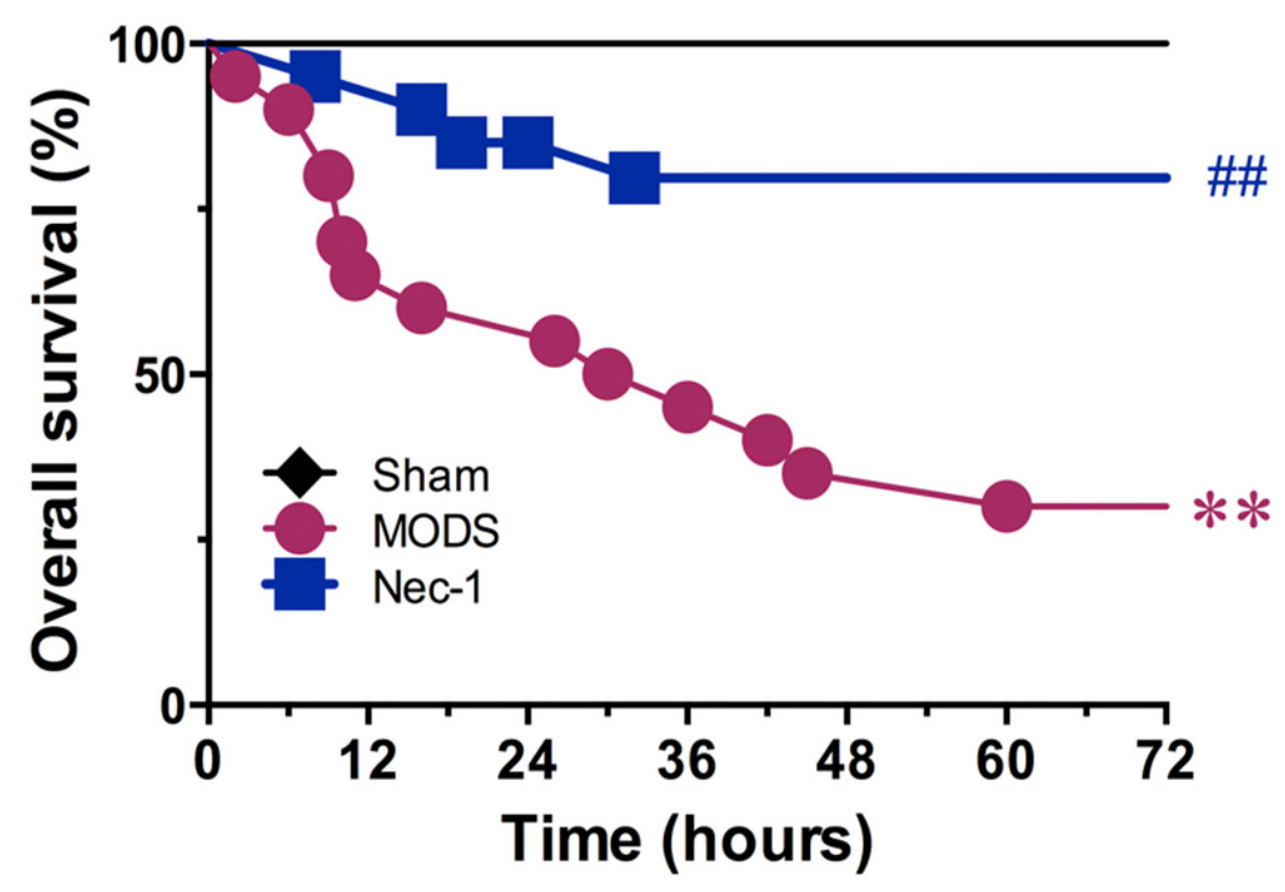

Figure 4: Effect of Nec-1 on MODS survival (25 animals per group). ${ }^{* *} p<0.01$ vs. the sham group, ${ }^{\#} p<0.05$ vs. the MODS group, and ${ }^{\# \#} p<0.01$ vs. the MODS group. 
A Time (hrs) 2

$\frac{\varepsilon}{\omega}$
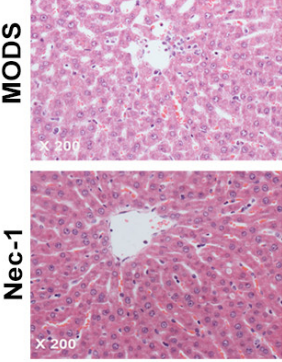

Time (hrs) 2
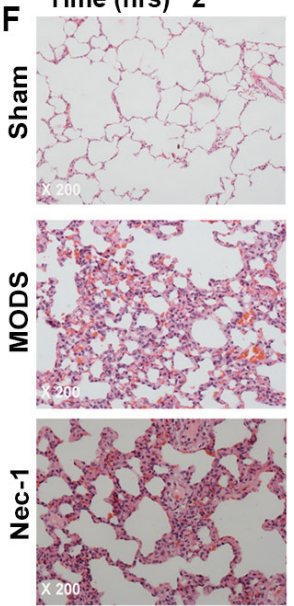

J Time (hrs) 2

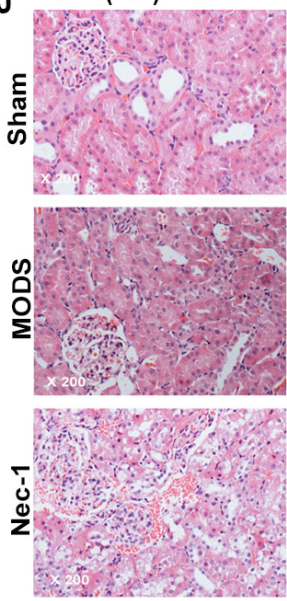

4
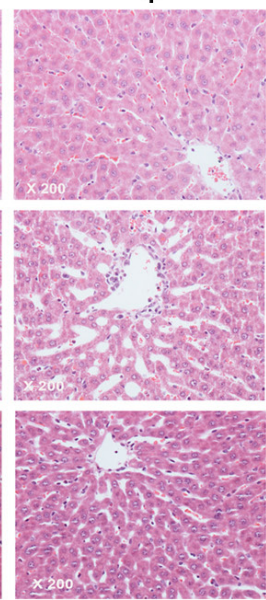

12
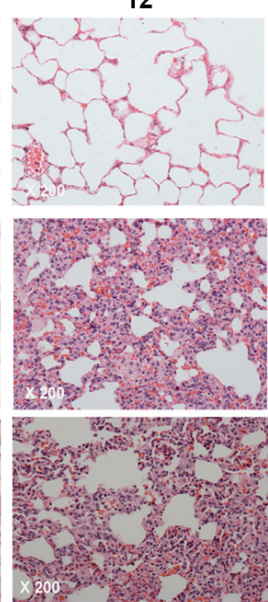

12

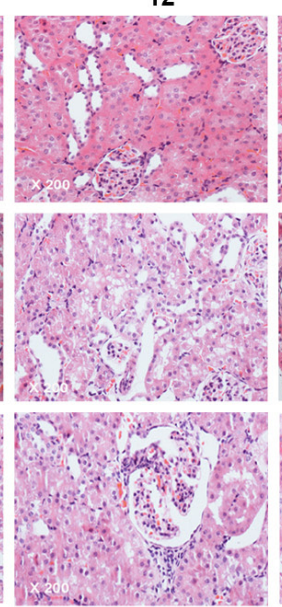

8
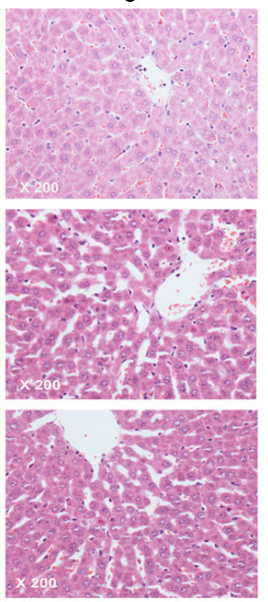

24
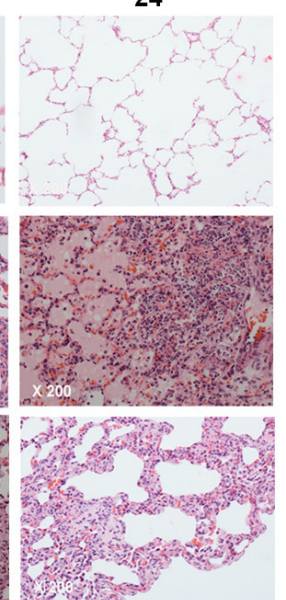

24
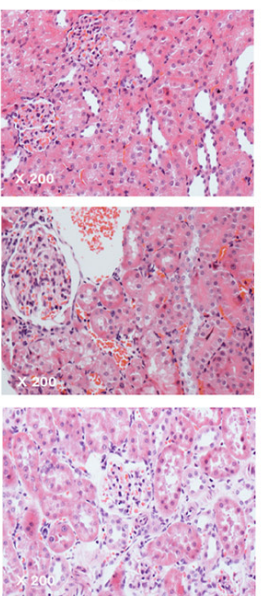

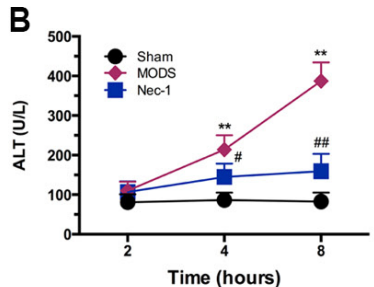

D

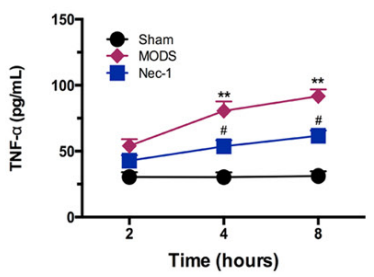

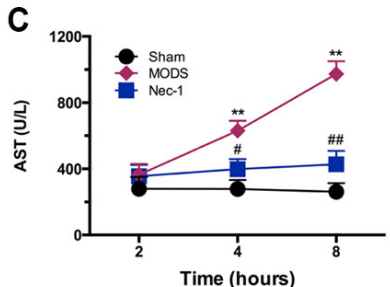

E

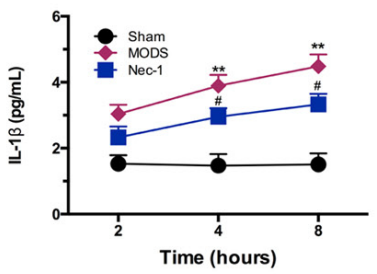

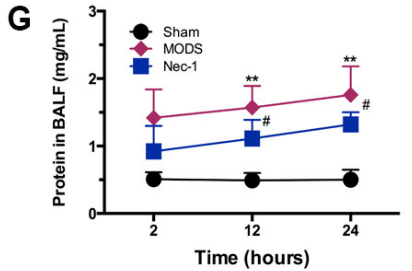
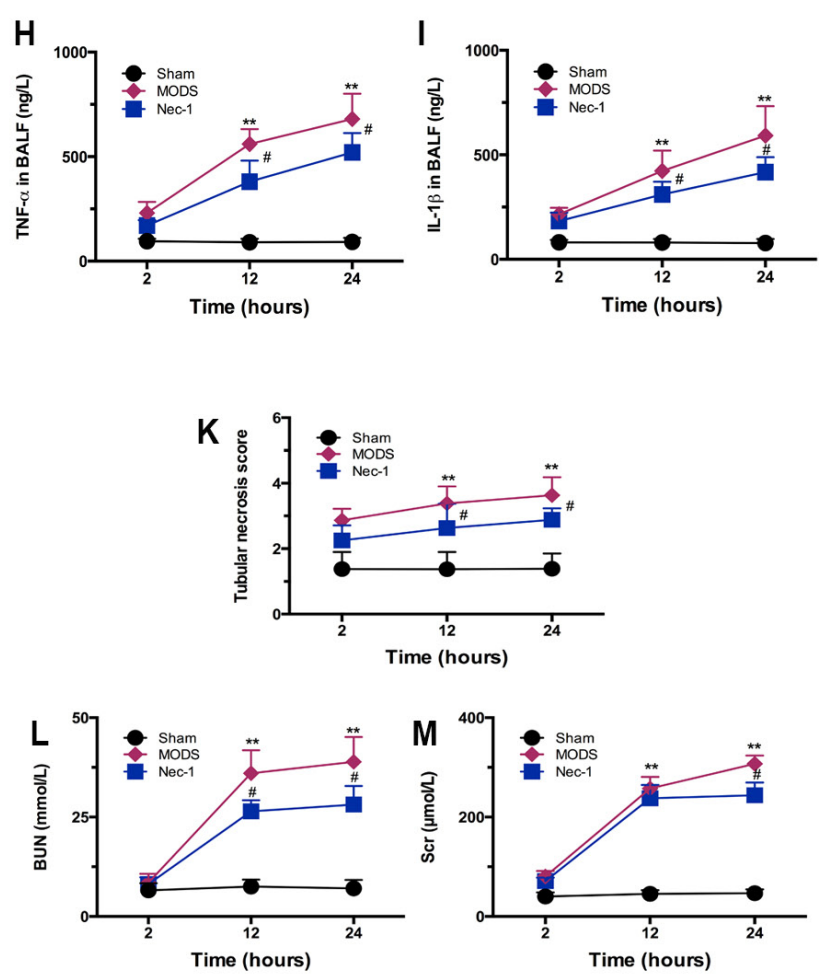

Figure 5: Nec-1 treatment significantly reduced necroptosis-induced multiorgan injury and dysfunction. Histological examination, biochemical analysis, and markers of systemic inflammation were assessed. Rats were sacrificed after inoculation, and organ tissues, serum, and BALF were obtained (A, F, and $\mathbf{J})$. Liver, lung, and kidney tissues were dissected and fixed in $4 \%$ paraformaldehyde. Paraffin-embedded tissue sections were stained with HE and examined by light microscopy. Images are representative of each group in three separate experiments (original magnification $\times 200$ ). (A) Liver; (F) lung; (J) and kidney tissue sections. (B-C, G, K-M) Liver, lung and kidney function markers in serum (mean \pm standard deviation of three separate experiments). (D and H) TNF- $\alpha$ in serum and BALF; (E and I) IL-1 $\beta$ in serum and BALF (mean \pm standard deviation of three separate experiments). ${ }^{* *} p<0.01$ vs. the sham group, ${ }^{*} p<0.05$ vs. the MODS group, and ${ }^{\# \#} p<0.01$ vs. the MODS group. 
Next, we examined organ injury and dysfunction by monitoring serum levels of organ injury-associated enzymatic indicators including ALT, AST, and the level of proteins in BALF, tubular necrotic score, BUN, and $\mathrm{SCR}$. We found that these enzymatic indicators were significantly elevated in MODS rats in comparison to the sham group. Interestingly, the time-dependent MODSinduced enzyme profile may indicate differences in the vulnerabilities of different organs to MODS. These indices of organ damage were significantly ameliorated by Nec-1 administration. In addition, the therapeutic effect of Nec-1 was also confirmed by histologic analysis. In liver, lung, and kidney samples, MODS-induced histopathologic changes, such as congestion, inflammatory cell infiltration, and degeneration, were ameliorated by Nec-1 administration. Although Nec-1 significantly reduced MODS-induced necroptosis, and organ damage, the function of several organs did not recover fully in Nec-1 treated rats. Therefore, early identification and immediate comprehensive treatment of MODS may improve outcomes.

The cellular mechanisms underlying the development and progression of MODS are complex, and of these multiple pathological processes the uncontrolled inflammatory response is known to contribute to rapid, progressive development of MODS [11-14]. Several studies have demonstrated that during early SIRS, the inflammatory cytokines TNF- $\alpha$ and IL- $1 \beta$ promote SIRS development [20-21]. Our results demonstrate that Nec-1 administration significantly inhibited MODSinduced elevations in TNF- $\alpha$ and IL- $1 \beta$ levels. Therefore, interfering with cytokine overproduction during early MODS may improve clinical outcomes. Importantly, Nec1 also improved survival in MODS rats. In the MODS group, the survival rate was reduced from $85 \%$ to $65 \%$ 24 hours, and from $35 \%$ to $80 \% 48$ hours, indicating that Nec-1 may be a potent and efficacious agent to treat MODS.

Measurement of the RIP3 substrate p-MLKL sheds light on the downstream mechanisms involved in executing necroptotic cell death [22-23]. We observed that $\mathrm{p}$-MLKL was strongly induced in MODS rats, while Nec-1 significantly suppressed MLKL pseudokinase at 12 hours. In addition, necroptosis is thought to directly trigger inflammation through necrotic cells' massive release of damage-associated molecular patterns, such as HMGB1
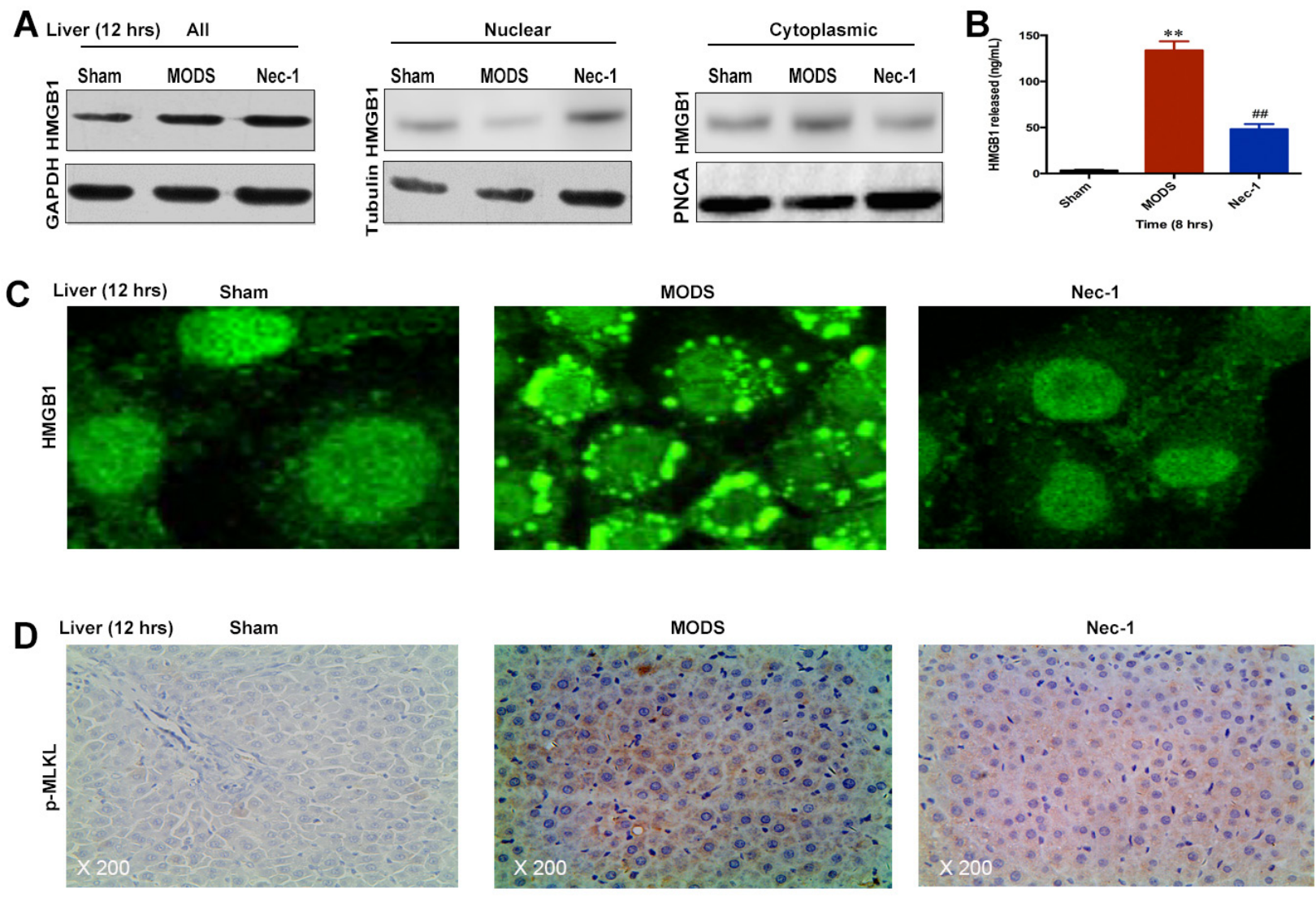

Figure 6: Nec-1 inhibits HMGB1 release and MLKL pseudokinase in rats with MODS. (A and C) HMGB1 protein expression was evaluated in liver tissues by western blotting and IF; (B) HMGB1 release was evaluated by ELISA. (D) p-MLKL expression in liver tissues was assessed by IHC staining. Data shown were representative images (original magnification $\times 200$ ) from three separate experiments. $* * p<0.01$ vs. the sham group and ${ }^{\#} p<0.01$ vs. the MODS group. 
[24]. In the present study, HMGB1 was found to be translocated from the nucleus to the cytoplasm in MODS rats, while Nec-1 treatment significantly suppressed HMGB1 translocation and reduced HMGB1 release in serum at 8 hours. Taken together, these results suggested that Nec-1 significantly inhibited HMGB1 release and MLKL pseudokinase.

Our conclusions are, however, limited by the scope of this study. First, there is no broad consensus on the molecular mechanisms and timing of cell death following MODS. This is in part because few of the pathways that influence cell death are understood, and because cell death form is typically very hard to assess following MODS. Second, it is unclear how necroptosis differs from nonprogrammed forms of necrosis. Necroptosis is a broad term, and is yet to be precisely defined. The factors triggering necroptosis are largely unknown, as are the factors allowing cells to escape death via this mechanism. It is also unclear how other intracellular and extracellular factors may contribute to necroptosis.

Additionally, the NIH now requires both genders be represented in animal research in order to preclude sex hormones interfere with the data. In fact, an increasing body of evidence from animal models has revealed that sex hormones play an intricate role in the pathological response to trauma-hemorrhage [25-26]. However, clinical studies have been unable to consistently reproduce these laboratory findings [27-28]. Therefore, the sex based outcome differences post-injury remain conflicting. In this study, male and female MODS rat was manifested in significantly more severe multiorgan injury and multiorgan dysfunction than sham surgery, and Nec-1 exerted a marked treatment effect by attenuating multiorgan injury and dysfunction, significantly improving survival of MODS rats.

In conclusion, considering the emerging significance of necroptosis in inflammatory disease, a better understanding of the pathological processes underlying necroptotic signaling will likely have important implications for the development of novel inflammatory disease therapies. This is the first study showing that necroptosis is involved the pathology of MODS, and further the first to describe the use of a necroptotic inhibitor, Nec-1, to treat MODS. Nec-1 may in the future be considered as a candidate adjunct treatment for MODS.

\section{MATERIALS AND METHODS}

\section{Ethics and statements}

This study was carried out in strict accordance with the recommendations in the Guide for the Care and Use of Laboratory Animals of the National Institutes of Health. The experiment protocols was approved by our Institutional Animal Care and Use Committee and the independent ethics committee at Tianjin Medical
University Cancer Institute \& Hospital, National Clinical Research Center for Cancer, Tianjin, China.

\section{Animal and establishing trauma/hemorrhagic shock model}

Six-week old male and female SD rats (200-250g), so that sex hormones would not interfere with the data, were obtained from the Experimental Animal Center, Academy of Military Medical Sciences, Beijing 100850, China (SCXK-2007-004). The rats were housed in a specific pathogen-free facility with free access to normal chow and water. The MODS rat model was successfully established as described previously [29-31]. Briefly, preconditioning was performed in a non-stressful, normothermic environment for more than 7 days prior to experiments. Rats were anesthetized by intraperitoneal injection of chloral hydrate $(200 \mathrm{mg} / \mathrm{kg})$. Afterward, a small incision was made, followed by catheterization in the right jugular vein and the right carotid artery for fluid resuscitation and bleeding, respectively.

An open mid-diaphyseal transverse fracture was created in the left femur to induce trauma, after which a mean arterial blood pressure of $30 \pm 5 \mathrm{mmHg}$ was maintained for 90 minutes through hemorrhage at $2.5 \mathrm{~mL} / 100 \mathrm{~g}$. Afterward, the rates were resuscitated for a period of 20 minutes with lactated Ringer's solution at a constant rate to manage the shock. The volume of the Ringer's solution was twice that of the blood loss. Finally, the catheters were removed, and the vessels were ligated. The mortality of experimental animals was about $30 \%$.

\section{Treatment design}

The experimental rats were randomly divided into three groups: (1) the sham group underwent the same anesthetic and surgical procedures and fluid resuscitation without induction of hemorrhage/trauma $(\mathrm{n}=25)$; (2) the MODS group was described above $(\mathrm{n}=25)$; and (3) the Nec-1 treatment group $(\mathrm{n}=25)$, and the Nec-1 was purchased by Sigma, MO, USA, in which the MODS rats were pretreatment and once daily with Nec-1 $(250 \mu \mathrm{g})$, which was diluted in resuscitation fluid and administered through the right jugular vein for about 20 minutes at a constant rate via a mini-pump. The dose of Nec-1 used in this study was based on previous study [11].

\section{Survival studies}

The first endpoint was to determine the effect of Nec-1 treatment on survival from MODS; rats were randomly divided into three experimental groups as mentioned previously ( $\mathrm{n}=25$ per group). All rats had free access to water and food and were frequently monitored by dedicated research personnel to determine the 72 hours survival statistics. 


\section{Fluorescence-activated cell sorting (FACS)}

The Annexin V-FITC (BD Pharmingen, CA, USA) and PI binding assay were performed to determine the apoptosis and necroptosis of multiorgan tissue cells, and single-cell suspensions were prepared and stained as previously described [32]. The single-cell suspensions were trypsinized, washed in PBS, and resuspended in binding buffer. The cells were incubated in the dark for 10 minutes with Annexin V-FITC $(100 \mathrm{ng} / \mathrm{ml})$ and 10 $\mathrm{ml}$ PI added to each group. Positive Annexin V staining indicated apoptosis, while positive PI and Annexin-V negative indicated necroptosis (necrosis). For each group, 500,000 events for single-cell suspensions were acquired and the frequency of positive cells was measured. Then examined using flow cytometer (FCM, BD FACS Array, San Jose, USA).

\section{Protein extraction and western blotting (WB)}

The multiorgan tissues were crushed in the dish, and the total, nucleus, and cytoplasm proteins were extracted by a cell lysate that was prepared containing an inhibitor cocktail against proteases and phosphatases. The protein concentration was measured by the BCA protein assay kit (Thermo Scientific, Rockford, Ill., USA). Equivalent amounts of protein were separated by $15 \%$ SDS-PAGE gel and transferred to a PVDF membrane at $4^{\circ} \mathrm{C}$. The membrane was then incubated overnight with rabbit anti-mouse polyclonal RIP1 (\#ab72139, abcam), RIP3 (\#ab152130, abcam), MLKL (\#5870, Sigma), highmobility group box 1 (HMGB1,\#ab18256, abcam), Tubulin (\#ab6046, abcam), PNCA (\#ab18197, abcam), and GAPDH (\#ab8245, abcam, Cambridge, UK), respectively. Then, membranes were incubated with HRP-conjugated secondary antibody for one hour at room temperature. Antibody binding was detected using the electrochemiluminescence (ECL) detection kit to produce a chemiluminescence signal, which was captured on X-ray film. Relative protein expression levels were calculated relative to GAPDH.

\section{Enzyme-linked immunosorbent assay (ELISA)}

The blood serum and bronchoalveolar lavage fluid (BALF) samples from rats were centrifuged at $3000 \mathrm{r} / \mathrm{min}$ for 5 minutes at $4^{\circ} \mathrm{C}$, and the separated serum samples were stored at $-20^{\circ} \mathrm{C}$ until use for assay. The ALT, AST, BUN, SCR, TNF- $\alpha$, IL-1 $\beta$, protein, and HMGB1 levels were measured using the monoclonal anti-rat capture antibody (R\&D Systems, Minneapolis, MN, USA) according to the manufacturer's instructions. Calibration curves were established, and each microtiter plate with a standard curve. The colorimetric reaction was read with a Benchmark Microplate Reader (Benchmark Electronics, Inc., Angleton, TX, USA).

\section{HE staining and immunohistochemical (IHC) assay}

The multiorgan tissues of rats from the different groups were collected and fixed with $10 \%$ neutral formaldehyde solution for 24 hours. The sample were dehydrated by a tissue-dehydrating machine, embedded in paraffin and sectioned into $4 \mu \mathrm{m}$ thick slices. Following roasting, slicing and dewaxing, routine hematoxylineosin staining was conducted and the sections were observed under light microscopy using Olympus BX40F microscope (Olympus Melville, NY, USA). Images were captured with a Sony $3 \mathrm{CCD}$ color video camera (Sony, Tokyo, Japan).

The expression of p-MLKL in liver tissues of three experimental group rats were assessed by three independent researchers who were blinded to followup data. Their conclusions were in complete agreement for $85 \%$ of cases indicating that this scoring method was highly reproducible. If two or all three agreed with the scoring results, the value was selected. If the results were completely different, then the pathologists worked collaboratively to confirm the score.

Briefly, the liver tissues from rats were dissected out, and tissue sections at $4 \mu \mathrm{m}$ were rehydrated, treated with $3 \% \mathrm{H}_{2} \mathrm{O}_{2}$ and blocked with $3 \%$ BSA. Subsequently, the tissue sections were incubated with anti-p-MLKL at Ser345 antibody (\#ab208910, abcam, 1:500), or with control IgG overnight at $4{ }^{\circ} \mathrm{C}$. The bound antibodies were detected with HRP-conjugated second antibodies and $\mathrm{DAB}$, followed by imaging under a light microscope. For evaluation of p-MLKL staining, a semi-quantitative scoring criterion was used, in which both staining intensity and positive areas were recorded. A staining index (values $0-16$ ) obtained as the intensity of positive staining (week, 1; moderate low, 2; moderate high, 3; strong, 4) and the proportion of immune-positive cells of interest $(0 \%$, $0 ;<10 \%, 1 ; 10-50 \%, 2 ; 51-80 \%, 3 ;>80 \%$, 4) were calculated.

\section{Immunofluorescence (IF) staining}

To confirm HMGB1 were translocated from the nucleus to the cytoplasm, we measured HMGB1 using IF staining. IF staining was briefly conducted on frozen section of liver tissues by HMGB1 (\#ab18256, abcam, 1:500). The specimens were examined with an inverted fluorescence microscope (IX50, Olympus, Japan).

\section{Statistical analyses}

Continuous variables were expressed as the mean \pm standard deviation (SD), and these were compared using an unpaired Student's T-test and one-way ANOVA. Overall survival curves were estimated using KaplanMeier analysis and compared using the stratified 
log-rank test. The differences with $p<0.05$ (two-tailed) were considered statistically significant. Data were analyzed using the statistical software Intercooled Stata version 8.2 for Windows (Stata Corporation, College Station, Texas, USA).

\section{Abbreviations}

DS: multiple organ dysfunction syndrome; Nec1: necrostatin-1; SD: Sprague-Dawley; SIRS: systemic inflammatory response syndrome; RIP1: receptorinteracting protein kinase 1; RIP3: receptor-interacting protein kinase 3; MLKL: mixed-lineage kinase domainlike protein; BALF: bronchoalveolar lavage fluid; TNF- $\alpha$ : tumor necrosis factor- $\alpha$; IL-1 $\beta$ : interleukin- $1 \beta$; ALT: alanine transaminase; AST: aspartate transaminase; BUN: blood urea nitrogen; SCR: serum creatinine.

\section{Author contributions}

Cui YL: Carried out conception and design, performed experimental study, collection and assembly of data, data analysis and interpretation, manuscript writing. Qiu LH, Zhou SY, and Li LF: performed experimental study, collection and assembly of data, data analysis and interpretation. Qian ZY and Liu XM: collection and assembly of data, data analysis and interpretation. Zhang HL, Ren XB, and Wang YQ: Carried out conception and design, collection and assembly of data, data analysis and interpretation, manuscript writing. All authors read and approved the final manuscript.

\section{ACKNOWLEDGMENTS}

We thank Professor Mao-Bin Meng, Dr. Bin Wang, Dr. Li-Ya Zhang, and the anonymous referee for his/her very helpful comments, which remarkably improved the quality of this paper.

\section{CONFLICTS OF INTEREST}

The manuscript describes original research and has not been submitted or is under consideration for publication in another journal. We also confirm that all the listed authors have participated actively in the study and have seen and approved the submitted manuscript. The authors do not have any possible conflicts of interest.

\section{FUNDING}

This work was supported in part by the National Natural Science Foundation of China (81301624), the Foundation of Tianjin Heath and Family Planning Commission (14KG101 and 2014KR07), and the National
Clinical Key Specially Project Foundation of the Ministry of Health in China (2011-873).

\section{REFERENCES}

1. Angus DC, Lindz-Zwirble WT, Lidicker J, Clermont G, Carcillo J, Pinsky MR. Epidemiology of severe sepsis in the United States: analysis of incidence, outcome, and associated costs of care. Crit Care Med. 2001; 29:1303-1310.

2. Beal AL, Cerra FB. Multiple organ failure syndrome in the 1990s. Systemic inflammatory response and organ dysfunction. JAMA. 1994; 271:226-233.

3. Marshall JC. Inflammation, coagulopathy, and the pathogenesis of multiple organ dysfunction syndrome. Crit Care Med. 2001; 29:S99-S106.

4. Seely AJ, Christou NV. Multiple organ dysfunction syndrome: exploring the paradigm of complex nonlinear systems. Crit Care Med. 2000; 28:2193-2200.

5. Wang $\mathrm{H}, \mathrm{Ma} \mathrm{S}$. The cytokine storm and factors determining the sequence and severity of organ dysfunction in multiple organ dysfunction syndrome. Am J Emerg Med. 2008; 26:711-715.

6. Bone RC, Balk RA, Cerra FB, Dellinger RP, Fein AM, Knaus WA, Schein RM, Sibbald WJ. Definitions for sepsis and organ failure and guidelines for the use of innovative therapies in sepsis. The ACCP/SCCM Consensus Conference Committee. American College of Chest Physicians/Society of Critical Care Medicine. Chest. 1992; 101:1644-1655.

7. Sapan HB, Paturusi I, Jusuf I, Patellongi I, Massi MN, Pusponegoro AD, Arief SK, Labeda I, Islam AA, Rendy L, Hatta M. Pattern of cytokine (IL-6 and IL-10) level as inflammation and anti-inflammation mediator of multiple organ dysfunction syndrome (MODS) in polytrauma. Int $\mathrm{J}$ Burns Trauma. 2016; 6:37-43.

8. Rosenthal MD, Moore FA. Persistent inflammation, immunosuppression, and catabolism: evolution of multiple organ dysfunction. Surg Infect. 2016; 17:167-172.

9. Gustot T. Multiple organ failure in sepsis: prognosis and role of systemic inflammatory response. Curr Opin Crit Care. 2011; 17:153-159.

10. Linkermann A, Green DR. Necroptosis. N Engl J Med. 2014; 370:455-465.

11. Duprez L, Takahashi N, Van Hauwermeiren F, Vandendriessche B, Goossens V, Vanden Berghe T, Declercq W, Libert C, Cauwels A, Vandenabeele P. RIP kinasedependent necrosis drives lethal systemic inflammatory response syndrome. Immunity. 2011; 35:908-918.

12. Ofengeim D, Yuan J. Regulation of RIP1 kinase signaling at the crossroads of inflammation and cell death. Nat Rev Mol Cell Biol. 2013; 14:727-736.

13. Pasparakis M, Vandenabeele P. Necroptosis and its role in inflammation. Nature. 2015; 517:311-320. 
14. Newton K, Manning G. Necroptosis and inflammation. Annu Rev Biochem. 2016; 85:743-763.

15. Linkermann A, Brasen JH, Darding M, Jin MK, Sanz AB, Heller JO, De Zen F, Weinlich R, Ortiz A, Walczak H, Weinberg JM, Green DR, Kunzendorf U, Krautwald S. Two independent pathways of regulated necrosis mediate ischemia-reperfusion injury. Proc Natl Acad Sci U S A. 2013; 110:12024-12029.

16. Linkermann A, Brasen JH, Himmerkus N, Liu S, Huber TB, Kunzendorf U, Krautwald S. Rip1 (receptor-interacting protein kinase 1) mediates necroptosis and contributes to renal ischemia/reperfusion injury. Kidney Int. 2012; 81:751-761.

17. Degterev A, Hitomi J, Germscheid M, Ch'en IL, Korkina O, Teng X. Identification of RIP1 kinase as a specific cellular target of necrostatins. Nat Chem Biol. 2008; 4:313-321.

18. Kopalli SR, Kang TB, Koppula S. Necroptosis inhibitors as therapeutic targets in inflammation mediated disorders a review of the current literature and patents. Expert Opin Ther Pat. 2016; 26:1239-1256.

19. Pasparakis M, Vandenabeele P. Necroptosis and its role in inflammation. Nature. 2015; 517:311-320.

20. Hack CE, Aarden LA, Thijs LG. Role of cytokines in sepsis. Adv Immunol. 1997; 66:101-195.

21. Adrie C, Pinsky MR. The inflammatory balance in human sepsis. Intensive Care Med. 2000; 26:364-375.

22. Vandenabeele P, Declercq W, Van Herreweghe F, Vanden Berghe T. The role of the kinases RIP1 and RIP3 in TNFinduced necrosis. Sci Signal. 2010; 3:re4.

23. Sun L, Wang H, Wang Z, He S, Chen S, Liao D, Wang L, Yan J, Liu W, Lei X, Wang X. Mixed lineage kinase domainlike protein mediates necrosis signaling downstream of RIP3 kinase. Cell. 2012; 148:213-227.
24. Kaczmarek A, Vandenabeele P, Krysko DV. Necroptosis: the release of damage-associated molecular patterns and its physiological relevance. Immunity. 2013; 38:209-223.

25. Trentzsch H, Nienaber U, Behnke M, Lefering R, Piltz S. Female sex protects from organ failure and sepsis after major trauma haemorrhage. Injury. 2014; 45:S20-S28.

26. Choudhry MA, Bland KI, Chaudry IH. Gender and susceptibility to sepsis following trauma. Endocr Metab Immune Disord Drug Targets. 2006; 6:127-135.

27. Gannon CJ, Pasquale M, Tracy JK, McCarter RJ, Napolitano LM. Male gender is associated with increased risk for postinjury pneumonia. Shock. 2004; 21:410-414.

28. Coimbra R, Hoyt DB, Potenza BM, Fortlage D, Hollingsworth-Fridlund P. Does sexual dimorphism influence outcome of traumatic brain injury patients? The answer is no! J Trauma. 2003; 54:689-700.

29. Zhang Y, Yao HP, Huang FF, Wu W, Gao Y, Chen ZB, Liang ZY, Liang TB. Allicin, a major component of garlic, inhibits apoptosis in vital organs in rats with trauma/hemorrhagic shock. Crit Care Med. 2008; 36:3226-3232.

30. Zhang Y, Liang L, Wu W, Gao Y, Chen ZB, Liang ZY, Liang TB. Resuscitation with hydroxyethyl starch solution prevents CD4+ T-lymphocyte apoptosis and modulates the balance of $\mathrm{T}$ helper type 1 and $\mathrm{T}$ helper type 2 responses in the rat with traumatic virgule/shill hemorrhagic shock. Shock. 2008; 30:692-698.

31. Zhang Y, Zhang J, Xu T, Wu W, Huang FF, Yu WQ, Zhang SY, Liang TB. Allicin ameliorates intraintestinal bacterial translocation after trauma/hemorrhagic shock in rats: the role of mesenteric lymph node dendritic cell. Surgery. 2016; 26:1239-1256.

32. Leong KG, Hu X, Li L, Noseda M, Larrivée B, Hull C, Hood L, Wong F, Karsan A. Activated Notch4 inhibits angiogenesis: role of beta 1-integrin activation. Mol Cell Biol. 2002; 22:2830-2841. 